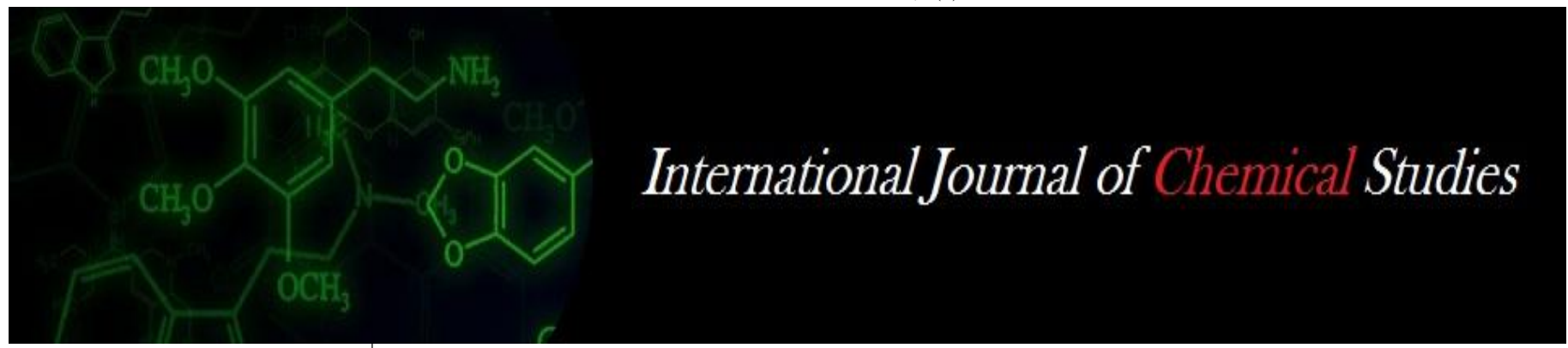

P-ISSN: 2349-8528

E-ISSN: 2321-4902

IJCS 2020; 8(1): 1887-1890

(C) 2020 IJCS

Received: 10-11-2019

Accepted: 12-12-2019

\section{Mahesh M}

Assistant Professor of Plant

Pathology, College of Sericulture,

Chintamani, UAS, Bangalore,

Karnataka, India

\section{Saifulla}

Professor (Retd.), Department of Plant Pathology, College of Agriculture, UAS, GKVK, Bangalore, Karnataka, India

Venkataravana $P$

Dean (Seri), College of

Sericulture, Chintamani, UAS

Bangalore, Karnataka, India

\section{Corresponding Author:}

Mahesh M

Assistant Professor of Plant Pathology, College of Sericulture,

Chintamani, UAS, Bangalore, Karnataka, India

\section{Identification of strains of Fusarium udum through host differential studies}

\author{
Mahesh M, M Saifulla and Venkataravana P
}

DOI: https://doi.org/10.22271/chemi.2020.v8.i1ab.8541

\begin{abstract}
Pathogenic behaviour of all the forty one isolates of $F$. udum on six pigeonpea host differentials indicated the existence of at least four strains in $F$. udum. Most of the $F$. udum isolates showed virulence reaction on cultivars TTB-7 and Bahar, whereas none of the isolates showed virulence on ICP 8863 and ICP 9174. Based on wilt reaction on six pigeonpea host differential lines, forty one $F$. udum isolates collected from major pigeonpea growing areas were categorised into four strains. Strain I included nine isolates viz., APFu-7, APFu-9, KFu-10, MSFu-1, MSFu-2, MSFu-3, MSFu-4, TNFu-2, and TNFu-5, and they were grouped as least virulant. Strain II comprised of fifteen isolates viz., APFu-1, APFu-4, APFu-5, APFu-6, APFu-8, APFu-10, APFu-11, KFU-6, KFU-7, KFU-8, KFU-9, KFU-12, TNFu-1, TNFu-3 and TNFu-4 and were grouped as moderately virulant. Strain III consisted of twelve isolates viz., APFu-2, APFu-3, BFu-1, KFU-4, KFU-5, KFU-11, KFU-13, KFU-15, KFU-16, MPFu-1, NDFu-1 and WBFu-1 and they were grouped as more virulant. Strain IV comprised of five isolates viz., KFU-1, KFU-2, KFU3, KFU-14 and UPFu-1 and they were grouped as most virulent.
\end{abstract}

Keywords: Pigeonpea, strain, host differential, Fusarium udum

\section{Introduction}

Pigeonpea (Cajanus cajan (L.) Millsp.) is a predominant pulse crop of India particularly in Karnataka. It is attacked by many diseases. Among the diseases, wilt caused by Fusarium udum Butler, is the most destructive one causing considerable yield loss.

The pathogen is primarily a soil inhabitant, hence controlling the disease is very difficult as no effective chemicals are available at present, even though application of carbendazim has been successful in controlling the disease, but to a limited extent and also it is not economical. The development of resistant varieties and combined application of bioagents and fungicides is considered as more practicable. However, developing resistant varieties is a tedious and time consuming procedure. Though several attempts have been made to develop resistant varieties, as ICRISAT developed wilt resistant variety "ICP 8863" and released by the name "Maruthi". Recently, a disease incidence up to 10 per cent in some locations is common. This may be due to the prevalence or development of new strains of $F$. udum in various geographical locations in India.

Presently, the information on the detection or identification of $F$. udum races/strains in the world in general and more particularly in India is lacking. Keeping this in view investigations were carried out on identification of $F$. udum strains using standard set of pigeonpea host differentials.

\section{Material and Methods}

Identification of Fusarium udum strains/variant through host differential reaction. A standard set of host differentials viz., ICP 8863, ICP 9174, C-11, BDN-1, TTB-7 and Bahar etc., supplied by IIPR, Kanpur were utilized for the identification of F. udum strains.

Giant culture of all the forty one $F$. udum isolates were prepared as mentioned earlier under 3.3. The seeds of all the six host differential lines were surface sterilized using two per cent sodium hypochlorite solution for one minute. The seeds were sown in $3 \times 3$ sq. $\mathrm{m}$. RCC pots containing $95 \mathrm{~kg}$ of red sandy loam soil sterilized with five per cent formalin solution. Artificially, five $\mathrm{kg}$ of giant culture was applied to the pots 10 days prior to sowing. Twenty seeds of each host differential lines were sown in $3 \times 3$ sq. m. RCC pots. 
Each treatment was replicated thrice. Observations were recorded at fortnight interval on symptom expression and no. of wilted plants upto 120 days after sowing. Symptoms started appearing after 20-30 days of sowing (Nene and Kannaiyan, 1982) ${ }^{[4]}$. Finally the strains were identified based on the differential reactions of the host for the pathogenic isolates as mentioned above. Reaction of the host differentials on $F$. udum isolates were recorded based on the scale developed by AICRP on Pigeonpea (Anon, 2007) ${ }^{[1]}$.

Based on wilt incidence on host differentials, the $41 \mathrm{~F}$. udum isolates were categorised into following four groups.

\begin{tabular}{|c|c|c|}
\hline Reaction & Wilt incidence & Strain/race \\
\hline Resistant & $: 0-10$ per cent & Least virulant (Strain I) \\
\hline Moderately resistant & $: 10.1-30$ per cent & $\begin{array}{c}\text { Moderately virulant (Strain } \\
\text { II) }\end{array}$ \\
\hline $\begin{array}{c}\text { Moderately } \\
\text { susceptible }\end{array}$ & $: 30.1-50$ per cent & More virulant (Strain III) \\
\hline Susceptible & $\begin{array}{c}:>50-100 \text { per } \\
\text { cent }\end{array}$ & Most virulant (Strain IV) \\
\hline
\end{tabular}

\section{Results and Discussion}

Identification of Fusarium udum strains through host pigeonpea differential reactions

An attempt was made to differentiate the F. udum isolates based on host differential reactions by employing six pigeonpea genotypes differing in their susceptibility against wilt in glass house studies. Wilt incidence and reactions of six pigeonpea wilt host differentials viz., ICP 8863, ICP 9174, C11, BDN-1, TTB-7 and Bahar against forty one F. udum isolates are presented in Table-1 and Table-2.

Based on wilt incidence, the host differentials were categorised into four groups viz., resistant (0-10\%), moderately resistant (10.1-30\%), moderately susceptible $(30.1-50 \%)$ and susceptible (>50\%) (Table-1). Investigations carried out in glass house revealed that ICP 8863 and ICP 9174 were resistant to all the forty one isolates, on the contrary TTB-7 and Bahar were susceptible to most of the isolates. Whereas $\mathrm{C}-11$ showed resistant reaction to thirty six isolates viz., $\mathrm{APFu}-1, \mathrm{APFu}-2, \mathrm{APFu}-3, \mathrm{APFu}-4, \mathrm{APFu}-5$, APFu-6, APFu-7, APFu-8, APFu-9, APFu-10, APFu-11, BFu-1, KFU-4, KFU-5, KFU-6, KFU-7, KFU-8, KFU-9, KFu-10, KFU-11, KFU-12, KFU-13, KFU-15, KFU-16, MPFu-1, MSFu-1, MSFu-2, MSFu-3, MSFu-4, NDFu-1, TNFu-1, TNFu-2, TNFu-3, TNFu-4, TNFu-5 and WBFu-1. However, it showed moderately resistant reaction to five isolates viz., KFU-1, KFU-2, KFU-3, KFU-14 and UPFu-1, whereas BDN-1 showed resistant reaction to twenty four isolates viz., APFu-1, APFu-4, APFu-5, APFu-6, APFu-7, APFu-8, APFu-9, APFu-10, APFu-11, KFU-6, KFU-7, KFU8, KFU-9, KFu-10, KFU-12, MSFu-1, MSFu-2, MSFu-3,
MSFu-4, TNFu-1, TNFu-2, TNFu-3, TNFu-4 and TNFu-5 and also it showed moderately resistant reaction to seventeen isolates viz., APFu-2, APFu-3, BFu-1, KFU-1, KFU-2, KFU3, KFU-4, KFU-5, KFU-11, KFU-13, KFU-14, KFU-15, KFU-16, MPFu-1, NDFu-1, UPFu-1 and WBFu-1 (Table-2). Six pigeonpea host differential lines were evaluated against forty one $F$. udum isolates. Based on wilt incidence and reaction, $F$. udum isolates were categorised into four strains (Table 3). Strain I includes nine isolates viz., APFu-7, APFu9, KFu-10, MSFu-1, MSFu-2, MSFu-3, MSFu-4, TNFu-2, and TNFu-5, on which five differentials viz., ICP 8863, ICP 9174, C-11, BDN-1 and Bahar recorded 0-10\% wilt incidence and showed resistant reaction TTB-7 differential recorded $10.1-30 \%$ wilt incidence and showed moderately resistant reaction and they were grouped as least virulent strain. Strain II comprised of fifteen isolates viz., APFu-1, APFu-4, APFu5, APFu-6, APFu-8, APFu-10, APFu-11, KFU-6, KFU-7, KFU-8, KFU-9, KFU-12, TNFu-1, TNFu-3 and TNFu-4 on which four differential lines viz., ICP 8863, ICP 9174, C-11 and $\mathrm{BDN}-1$ recorded $0-10$ per cent wilt incidence and showed resistant reaction, while TTB-7 and Bahar recorded 30.1-50 per cent wilt incidence and showed moderately susceptible reaction and they were grouped as moderately virulent strain. Strain III consisted of twelve isolates viz., APFu-2, APFu-3, BFu-1, KFU-4, KFU-5, KFU-11, KFU-13, KFU-15, KFU-16, MPFu-1, NDFu-1 and WBFu-1 on which three differential lines viz., ICP 8863, ICP 9174 and C-11 recorded 0-10 per cent wilt incidence and showed resistant reaction, but BDN-1 recorded 10.1-30 per cent wilt incidence and showed moderately resistant reaction, whereas TTB-7 and Bahar recorded $>50$ per cent wilt incidence and showed susceptible reaction and they were grouped as more virulent strain. Strain IV comprised of five isolates viz., KFU-1, KFU-2, KFU-3, KFU-14 and UPFu-1 on which ICP 8863 and ICP 9174 recorded 0-10 per cent wilt incidence and showed resistant reaction, while $\mathrm{C}-11$ and $\mathrm{BDN}-1$ recorded $10.1-30$ per cent wilt incidence and showed moderately resistant reaction, whereas TTB-7 and Bahar recorded $>50$ per cent wilt incidence and showed susceptible reaction and they were grouped as most virulent strain.

Pathogenic variation in Fusarium is well demonstrated by Colina et al. (1985) ${ }^{[2]}$; Haware and Nene (1982) ${ }^{[4]}$. Phillips (1988) ${ }^{[5]}$ demonstrated pathogenic variation in $F$. oxysporum f.sp. ciceri, whereas, Shit and Sengupta (1980) ${ }^{[9]}$; Sataraddi (1998) ${ }^{[7]}$; Reddy et al. (1999) ${ }^{[6]}$ and Shashi Misra and Vishwa Dhar (2003) ${ }^{[8]}$ suggested the possibility of existence of pathogenic races of the fungus as indicated by the differential response of the same variety under different conditions observed variability in the isolates of Fusarium spp.

Table 1: Development of wilt disease by Fusarium udum isolates collected from different geographical locations in India on pigeonpea wilt host differentials

\begin{tabular}{|c|c|c|c|c|c|c|c|}
\hline \multirow{2}{*}{ Sl. no. } & \multirow{2}{*}{ Isolates } & \multicolumn{7}{|c|}{ Wilt incidence against host differentials } \\
\cline { 3 - 8 } & & ICP 8863 & ICP 9174 & C-11 & BDN-1 & TTB-7 & Bahar \\
\hline 1 & APFu-1 & 0.00 & 9.09 & 0.00 & 9.09 & 38.00 & 36.45 \\
\hline 2 & APFu-2 & 0.00 & 7.69 & 7.69 & 14.11 & 66.67 & 77.78 \\
\hline 3 & APFu-3 & 0.00 & 9.50 & 8.00 & 13.00 & 60.00 & 61.67 \\
\hline 4 & APFu-4 & 7.00 & 0.00 & 0.00 & 2.50 & 38.89 & 37.00 \\
\hline 5 & APFu-5 & 9.09 & 6.67 & 0.00 & 0.00 & 40.87 & 44.44 \\
\hline 6 & APFu-6 & 6.00 & 1.00 & 0.00 & 0.00 & 37.29 & 36.00 \\
\hline 7 & APFu-7 & 3.33 & 0.00 & 1.00 & 0.00 & 20.40 & 2.00 \\
\hline 8 & APFu-8 & 0.00 & 0.00 & 7.14 & 7.65 & 38.26 & 35.00 \\
\hline 9 & APFu-9 & 5.00 & 0.00 & 5.00 & 0.00 & 18.18 & 5.00 \\
\hline 10 & APFu-10 & 0.00 & 0.00 & 6.67 & 0.00 & 33.00 & 36.67 \\
\hline
\end{tabular}

$\sim 1888 \sim$ 


\begin{tabular}{|c|c|c|c|c|c|c|c|}
\hline 11 & APFu-11 & 3.33 & 4.30 & 7.14 & 3.04 & 35.00 & 33.00 \\
\hline 12 & BFu-1 & 5.00 & 0.00 & 5.00 & 20.00 & 53.85 & 52.00 \\
\hline 13 & KFu-1 & 2.09 & 6.67 & 15.38 & 17.69 & 90.00 & 84.00 \\
\hline 14 & KFu-2 & 9.00 & 8.11 & 25.00 & 12.22 & 80.00 & 66.67 \\
\hline 15 & KFu-3 & 0.00 & 6.67 & 18.00 & 20.00 & 65.00 & 57.14 \\
\hline 16 & KFu-4 & 5.00 & 0.00 & 5.00 & 20.00 & 58.57 & 56.00 \\
\hline 17 & KFu-5 & 0.00 & 0.00 & 3.00 & 18.00 & 63.33 & 80.00 \\
\hline 18 & KFu-6 & 2.22 & 0.00 & 2.00 & 1.00 & 35.00 & 31.11 \\
\hline 19 & KFu-7 & 5.00 & 2.00 & 2.50 & 0.00 & 32.00 & 37.69 \\
\hline 20 & KFu-8 & 7.69 & 0.00 & 0.00 & 9.09 & 35.00 & 36.67 \\
\hline 21 & KFu-9 & 3.08 & 8.11 & 0.00 & 2.00 & 40.00 & 32.50 \\
\hline 22 & KFu-10 & 8.18 & 0.00 & 0.00 & 0.00 & 18.52 & 5.00 \\
\hline 23 & KFu-11 & 2.50 & 7.14 & 8.00 & 15.00 & 60.00 & 70.00 \\
\hline 24 & KFu-12 & 2.00 & 6.67 & 0.00 & 9.09 & 45.00 & 37.00 \\
\hline 25 & KFu-13 & 3.11 & 4.76 & 5.00 & 5.00 & 56.15 & 80.00 \\
\hline 26 & KFu-14 & 8.18 & 0.00 & 16.00 & 18.33 & 77.83 & 86.36 \\
\hline 27 & KFu-15 & 0.00 & 0.00 & 3.33 & 15.00 & 62.50 & 54.55 \\
\hline 28 & KFU-16 & 2.50 & 3.08 & 6.67 & 12.50 & 75.00 & 57.14 \\
\hline 29 & MPFu-1 & 0.00 & 3.33 & 0.00 & 13.00 & 52.00 & 54.55 \\
\hline 30 & MSFu-1 & 0.00 & 0.00 & 2.00 & 4.25 & 12.00 & 5.00 \\
\hline 31 & MSFu-2 & 0.00 & 7.14 & 7.14 & 0.00 & 23.08 & 8.00 \\
\hline 32 & MSFu-3 & 0.00 & 0.00 & 6.25 & 2.50 & 14.29 & 5.00 \\
\hline 33 & MSFu-4 & 0.00 & 0.00 & 0.00 & 4.29 & 12.50 & 5.00 \\
\hline 34 & NDFu-1 & 0.00 & 6.25 & 5.00 & 12.00 & 60.00 & 53.00 \\
\hline 35 & TNFu-1 & 5.00 & 4.29 & 4.00 & 5.00 & 36.67 & 32.22 \\
\hline 36 & TNFu-2 & 4.29 & 1.11 & 9.09 & 0.00 & 22.22 & 6.67 \\
\hline 37 & TNFu-3 & 0.00 & 0.00 & 0.00 & 0.00 & 35.00 & 38.46 \\
\hline 38 & TNFu-4 & 3.57 & 4.11 & 0.00 & 9.09 & 33.53 & 36.36 \\
\hline 39 & TNFu-5 & 5.38 & 7.14 & 2.50 & 0.00 & 15.00 & 4.29 \\
\hline 40 & UPFu-1 & 3.09 & 0.00 & 17.50 & 16.67 & 60.00 & 54.55 \\
\hline 41 & WBFu-1 & 0.00 & 8.33 & 6.35 & 16.67 & 56.15 & 60.00 \\
\hline & & & & & & & \\
\hline & & & & & & \\
\hline
\end{tabular}

Table 2: Reaction of pigeonpea host differentials to the isolates of Fusarium udum collected from different geographical locations in India

\begin{tabular}{|c|c|c|c|c|c|c|c|}
\hline \multirow{2}{*}{ Sl. no. } & \multirow{2}{*}{ Isolates } & \multicolumn{6}{|c|}{ Wilt reaction against host differentials } \\
\hline & & ICP 8863 & ICP 9174 & C-11 & BDN-1 & TTB-7 & Bahar \\
\hline 1 & APFu-1 & $\mathrm{R}$ & $\mathrm{R}$ & $\mathrm{R}$ & $\mathrm{R}$ & MS & MS \\
\hline 2 & APFu-2 & $\mathrm{R}$ & $\mathrm{R}$ & $\mathrm{R}$ & MR & $S$ & $S$ \\
\hline 3 & APFu-3 & $\mathrm{R}$ & $\mathrm{R}$ & $\mathrm{R}$ & MR & $S$ & $S$ \\
\hline 4 & APFu-4 & $\mathrm{R}$ & $\mathrm{R}$ & $\mathrm{R}$ & $\mathrm{R}$ & MS & MS \\
\hline 5 & APFu-5 & $\mathrm{R}$ & $\mathrm{R}$ & $\mathrm{R}$ & $\mathrm{R}$ & MS & MS \\
\hline 6 & APFu-6 & $\mathrm{R}$ & $\mathrm{R}$ & $\mathrm{R}$ & $\mathrm{R}$ & MS & MS \\
\hline 7 & APFu-7 & $\mathrm{R}$ & $\mathrm{R}$ & $\mathrm{R}$ & $\mathrm{R}$ & MR & $\mathrm{R}$ \\
\hline 8 & APFu-8 & $\mathrm{R}$ & $\mathrm{R}$ & $\mathrm{R}$ & $\mathrm{R}$ & MS & MS \\
\hline 9 & APFu-9 & $\mathrm{R}$ & $\mathrm{R}$ & $\mathrm{R}$ & $\mathrm{R}$ & MR & $\mathrm{R}$ \\
\hline 10 & APFu-10 & $\mathrm{R}$ & $\mathrm{R}$ & $\mathrm{R}$ & $\mathrm{R}$ & MS & MS \\
\hline 11 & APFu-11 & $\mathrm{R}$ & $\mathrm{R}$ & $\mathrm{R}$ & $\mathrm{R}$ & MS & MS \\
\hline 12 & $\mathrm{BFu}-1$ & $\mathrm{R}$ & $\mathrm{R}$ & $\mathrm{R}$ & MR & $S$ & $\mathrm{~S}$ \\
\hline 13 & KFu-1 & $\mathrm{R}$ & $\mathrm{R}$ & MR & MR & $S$ & $S$ \\
\hline 14 & $\mathrm{KFu}-2$ & $\mathrm{R}$ & $\mathrm{R}$ & MR & MR & $\mathrm{S}$ & $S$ \\
\hline 15 & $\mathrm{KFu}-3$ & $\mathrm{R}$ & $\mathrm{R}$ & MR & MR & $S$ & $\mathrm{~S}$ \\
\hline 16 & KFu-4 & $\mathrm{R}$ & $\mathrm{R}$ & $\mathrm{R}$ & MR & $\mathrm{S}$ & $\mathrm{S}$ \\
\hline 17 & KFu-5 & $\mathrm{R}$ & $\mathrm{R}$ & $\mathrm{R}$ & MR & $S$ & $S$ \\
\hline 18 & KFu-6 & $\mathrm{R}$ & $\mathrm{R}$ & $\mathrm{R}$ & $\mathrm{R}$ & MS & MS \\
\hline 19 & KFu-7 & $\mathrm{R}$ & $\mathrm{R}$ & $\mathrm{R}$ & $\mathrm{R}$ & MS & MS \\
\hline 20 & $\mathrm{KFu}-8$ & $\mathrm{R}$ & $\mathrm{R}$ & $\mathrm{R}$ & $\mathrm{R}$ & MS & MS \\
\hline 21 & KFu-9 & $\mathrm{R}$ & $\mathrm{R}$ & $\mathrm{R}$ & $\mathrm{R}$ & MS & MS \\
\hline 22 & KFu-10 & $\mathrm{R}$ & $\mathrm{R}$ & $\mathrm{R}$ & $\mathrm{R}$ & MR & $\mathrm{R}$ \\
\hline 23 & KFu-11 & $\mathrm{R}$ & $\mathrm{R}$ & $\mathrm{R}$ & MR & $\mathrm{S}$ & $S$ \\
\hline 24 & $\mathrm{KFu}-12$ & $\mathrm{R}$ & $\mathrm{R}$ & $\mathrm{R}$ & $\mathrm{R}$ & MS & MS \\
\hline 25 & $\mathrm{KFu}-13$ & $\mathrm{R}$ & $\mathrm{R}$ & $\mathrm{R}$ & MR & $\mathrm{S}$ & $\mathrm{S}$ \\
\hline 26 & KFu-14 & $\mathrm{R}$ & $\mathrm{R}$ & MR & MR & $S$ & $S$ \\
\hline 27 & $\mathrm{KFu}-15$ & $\mathrm{R}$ & $\mathrm{R}$ & $\mathrm{R}$ & MR & $\mathrm{S}$ & S \\
\hline 28 & KFU-16 & $\mathrm{R}$ & $\mathrm{R}$ & $\mathrm{R}$ & MR & $\mathrm{S}$ & $\mathrm{S}$ \\
\hline 29 & MPFu-1 & $\mathrm{R}$ & $\mathrm{R}$ & $\mathrm{R}$ & MR & $S$ & $S$ \\
\hline 30 & MSFu-1 & $\mathrm{R}$ & $\mathrm{R}$ & $\mathrm{R}$ & $\mathrm{R}$ & MR & $\mathrm{R}$ \\
\hline 31 & MSFu-2 & $\mathrm{R}$ & $\mathrm{R}$ & $\mathrm{R}$ & $\mathrm{R}$ & MR & $\mathrm{R}$ \\
\hline 32 & MSFu-3 & $\mathrm{R}$ & $\mathrm{R}$ & $\mathrm{R}$ & $\mathrm{R}$ & MR & $\mathrm{R}$ \\
\hline 33 & MSFu-4 & $\mathrm{R}$ & $\mathrm{R}$ & $\mathrm{R}$ & $\mathrm{R}$ & MR & $\mathrm{R}$ \\
\hline
\end{tabular}




\begin{tabular}{|c|c|c|c|c|c|c|c|}
\hline 34 & NDFu-1 & R & R & R & MR & S & S \\
\hline 35 & TNFu-1 & R & R & R & R & MS & MS \\
\hline 36 & TNFu-2 & R & R & R & R & MR & R \\
\hline 37 & TNFu-3 & R & R & R & R & MS & MS \\
\hline 38 & TNFu-4 & R & R & R & R & MS & MS \\
\hline 39 & TNFu-5 & R & R & R & R & MR & R \\
\hline 40 & UPFu-1 & R & R & MR & MR & S & S \\
\hline 41 & WBFu-1 & R & R & R & MR & S & S \\
\hline
\end{tabular}

$\mathrm{R}=$ Resistant $(0-10 \%)$

$\mathrm{MR}=$ Moderately resistant (10.1-30.0)

MS= Moderately susceptible (30.1-50.0)

$\mathrm{S}=$ Susceptible $(>50.00)$

Table 3: Categorization of the isolates of Fusarium udum collected from different geographical locations in India into strains on the basis of host differential reactions

\begin{tabular}{|c|c|c|c|c|c|}
\hline \multirow[b]{2}{*}{$\begin{array}{l}\text { Sl. } \\
\text { no. }\end{array}$} & \multirow[b]{2}{*}{$\begin{array}{c}\text { Pigeonpea differential } \\
\text { line }\end{array}$} & \multicolumn{4}{|c|}{ Reaction of Isolates } \\
\hline & & $\begin{array}{c}\text { Strain I } \\
\text { (Least virulent) }\end{array}$ & $\begin{array}{c}\text { Strain II } \\
\text { (Moderately virulent) }\end{array}$ & $\begin{array}{c}\text { Strain III } \\
\text { (More virulent) }\end{array}$ & $\begin{array}{c}\text { Strain IV } \\
\text { (Most virulent) }\end{array}$ \\
\hline 1 & ICP 8863 & $\mathrm{R}$ & $\mathrm{R}$ & $\mathrm{R}$ & $\mathrm{R}$ \\
\hline 2 & ICP 9174 & $\mathrm{R}$ & $\mathrm{R}$ & $\mathrm{R}$ & $\mathrm{R}$ \\
\hline 3 & C-11 & $\mathrm{R}$ & $\mathrm{R}$ & $\mathrm{R}$ & MR \\
\hline 4 & BDN-1 & $\mathrm{R}$ & $\mathrm{R}$ & MR & MR \\
\hline 5 & TTB-7 & MR & MS & $\mathrm{S}$ & $\mathrm{S}$ \\
\hline 6 & Bahar & S & MS & $S$ & S \\
\hline & & $\begin{array}{c}\text { APFu-7 (Menavalu) APFu-9 } \\
\text { (Regadimylapuram) } \\
\text { KFu-10 (Konkanahalli) } \\
\text { MSFu-1 (Akola) } \\
\text { MSFu-2 (Chanai) } \\
\text { MSFu-3 (Badnapur) } \\
\text { MSFu-4 (Latur) } \\
\text { TNFu-2 (Kodavadi) } \\
\text { TNFu-5 (Thiru varankulam) }\end{array}$ & $\begin{array}{c}\text { APFu-1 (Appanpuram) } \\
\text { APFu-4 (Merakapudi) } \\
\text { APFu-5 (Kichvala) } \\
\text { APFu-6 (Mahadevanpet) } \\
\text { APFu-8 (Mannemkonda) } \\
\text { APFu-10 (Bandimidipalli) } \\
\text { APFu-11 (Thandur) } \\
\text { KFU-6 (Nimbala) } \\
\text { KFU-7 (Sirunuru) } \\
\text { KFU-8 (Uppalav) } \\
\text { KFU-9 (Kolluru) } \\
\text { KFU-12 (Kandali) } \\
\text { TNFu-1 (Coimbatore) } \\
\text { TNFu-3 (Vamban) } \\
\text { TNFu-4 (Thiruchi) }\end{array}$ & $\begin{array}{c}\text { APFu-2 (Inkallu) } \\
\text { APFu-3 (Kesanapalli) } \\
\text { BFu-1 (Muzaffarpur) } \\
\text { KFU-4 (Salotagi) } \\
\text { KFU-5 (Aland) } \\
\text { KFU-11 } \\
\text { (Margundanahalli) } \\
\text { KFU-13 (Ankapur) } \\
\text { KFU-15 (Beerihundi) } \\
\text { KFU-16 (Hidapanur) } \\
\text { MPFu-1 (JNKVV farm) } \\
\text { NDFu-1 (Zaidapur) } \\
\text { WBFu-1 (Murshidabad) }\end{array}$ & $\begin{array}{c}\text { KFU-1 (GKVK, } \\
\text { Bangalore) } \\
\text { KFU-2 (Byraganapura) } \\
\text { KFU-3 (Dhannampalya) } \\
\text { KFU-14 } \\
\text { (Chokkandahalli) } \\
\text { UPFu-1 (Kanpur) }\end{array}$ \\
\hline & Total no. of isolates & (09) & (15) & (12) & 05 \\
\hline
\end{tabular}

\section{References}

1. Anonymous, Annual Report for 2007-08. All India Coordinated Research Project on Pigeonpea, IIPR, Kanpur, 2007, 239.

2. Colina J, Cashs A, Diaz RM. Races of Fusarium oxysporum fsp Ciceri ICN Andalucia, Southern Spain. $1985 ; 13: 24-26$.

3. Haware MP, Nene YL. Races of Fusarium oxysporum f.sp. Ciceri. Plant Disease. 1982; 66:809-810.

4. Nene YL, Kannaiyan J. Screening of Pigeonpea for resistance to Fusarium wilt. Plant Disease. 1982; 66:306307.

5. Philips JC. A distinct race of chickpea wilt in California Inter. Chickpea Newls. 1988; 18:19-20.

6. Reddy MV, Raju TN, Lenne JM. Diseases of Pigeonpea. In: The Pathology of food and pasture legumes. (Eds. Allen DJ, Lenne JM), CAB International, Wallingford, United Kingdom. 1999; 750:517-558.

7. Sataraddi AR, Variability in Fusarium udum causing wilt of Pigeonpea. Ph.D. Thesis, Univ. Agri. Sci. Dharwad, 1998, 112.

8. Shashi Misra, Vishwadhar, Comparative conidial morphology and virulence of Fusarium udum from different pigeonpea varieties. Farm Sci. J, 12(2), 132134.
9. Shit SK, Sengupta PK. Possible existence of races of Fusarium oxysporum f.sp. Udum incident of the wilt of Pigeonpea. Indian J Agric. Sci. 1978-2003; 48:629-630. 